\title{
Link-Based Signalized Arterial Progression Optimization with Practical Travel Speed
}

\author{
Wu Xianyu, ${ }^{1}$ Hu Peifeng, ${ }^{2}$ and Yuan Zhenzhou ${ }^{1}$ \\ ${ }^{1}$ MOE Key Laboratory for Urban Transportation Complex Systems Theory and Technology, \\ Beijing Jiaotong University, Beijing 100044, China \\ ${ }^{2}$ Center for Advanced Transportation Education and Research (CATER), UNR, MS 258, Reno, NV 89557, USA \\ Correspondence should be addressed to Wu Xianyu; wuxy@bjtu.edu.cn
}

Received 17 June 2013; Revised 6 September 2013; Accepted 6 September 2013

Academic Editor: Mohamed Fathy El-Amin

Copyright (c) $2013 \mathrm{Wu}$ Xianyu et al. This is an open access article distributed under the Creative Commons Attribution License, which permits unrestricted use, distribution, and reproduction in any medium, provided the original work is properly cited.

\begin{abstract}
Bandwidth is defined as the maximum amount of green time for a designated movement as it passes through an arterial. In most previous studies, bandwidth has been referred to arterial bandwidth. In practice, a balance between link bandwidth and arterial bandwidth has proven to be important in optimizing coordinated signal timing plans, because not all drivers need to pass through all the intersections on an arterial. This study proposes an algorithm on how to obtain an optimal coordinated signal timing plan with both optimal link bandwidth and optimal arterial bandwidth considering practical vehicles' speed. The weighted link bandwidth attainability is introduced as an additional measure of effectiveness for assessing the optimization results. The link bandwidth optimization is built based on the improvement of Messer's algorithm about bandwidth optimization. The arterial bandwidth optimization algorithm takes into consideration the weighted link bandwidth attainability while selecting phase sequences. The proposed algorithm is demonstrated in a case study, and many improvements are archived when a balanced consideration is given to both link bandwidth and arterial bandwidth. Fine-tuning of initial signal timing plan is done using practical travel speed. The evaluation results show a rather significant improvement which is achieved.
\end{abstract}

\section{Introduction}

The objective of a signalized arterial progression is to provide continuous movement of vehicles and/or minimize the delay along an arterial. Bandwidth is defined as the maximum amount of green time for a designated movement as it passes through an arterial. It is an outcome of the signal timing plan that is determined by the offsets between intersections and the allotted green time for the coordinated phase at each intersection. Bandwidth (measured in seconds) can be defined in terms of two consecutive intersections (referred to as link bandwidth) or in terms of an entire arterial (referred to as arterial bandwidth). Bandwidth and with its associated measures of efficiency and attainability are measures that are often used to assess the effectiveness of a coordinated signal timing plan [1].

A larger progression bandwidth implies that more traffic on an arterial can progress through the signals without stops $[2,3]$. Furthermore, bandwidth-based signal timing is preferred because it better meets drivers' expectations. A signal timing solution without a good progression band, no matter how well it may claim to minimize system delays and stops, may not be acceptable to traffic engineers and travelers [4]. A study conducted by Yang indicated that bandwidthbased solutions generally outperform delay-based solutions based on several field studies [5].

From the late 1960s to the early 1980s, many researchers, including Little, Messer, and Brooks, made a significant progress and developed a series of bandwidth optimization algorithms [6]. Messer et al. developed a variable-sequence program to form the core of the PASSER software. They modified traditional green bandwidth method and added variable phase sequence operation in the software $[7,8]$. Little et al. developed the MAXBAND, using a more complicated mixed integer linear programming model [9]. Besides MAXBAND and PASSER, there are several other models that have been introduced. Gartner et al. developed MULTIBAND to improve the original MAXBAND [10]. In 1996, they 
published MULTIBAND-96 to further improve the model and applied to realistic networks [11]. Efforts to improve bandwidth-based optimization models and their computing algorithms continued up to the late 1990s [12-15]. Feng [16] developed the projection algorithm (PA) to optimize signal offsets and cycle length while considering start-up delay, residual queues, and unbalanced traffic volumes.

In these studies mentioned above, bandwidth is always defined in terms of an entire arterial. Arterial bandwidth is commonly used to describe capacity or maximized vehicle throughput. While arterial bandwidth is a good indicator for progression opportunities, it may not fully capture the overall arterial operation. For example, on an arterial with 10 signalized intersections, an arterial bandwidth solution allows vehicles to travel through the entire system. In reality, one must consider how many vehicles actually travel through all the intersections. In some cases, link bandwidth is more important than arterial bandwidth because of high volumes (especially in the case of high left-turn volumes) between some links. Therefore both link and arterial bandwidth should be considered in optimizing coordinated signal timing plans. In 2002, Gartner and Stamatiadis provided mathematical programming models for the development of optimal arterial-based progression schemes considering an individually weighted band that can be adapted to the prevailing traffic flows on that link [17]. Their models began to pay more attention to link bandwidth; however they did not consider the impact of speed variation and could not obtain the signal timing plan with both optimal arterial bandwidth and optimal link bandwidth. Although software tools, such as SYNCHRO and TRANSY7F, can depict both arterial bands and link bands, their optimization objective is network delay instead of bandwidth. A signal timing plan with both optimized link bandwidth and arterial bandwidth is normally difficult to be obtained [18]. To overcome the limitations of previous studies, this study focuses on an algorithm that can obtain a best signal timing plan with both optimal link bandwidth and optimal arterial bandwidth.

In an ideal coordinated system with optimal bandwidth, platoons with desired travel speed from an upstream intersection at the start of green should arrive at downstream intersection near start of the green indication. In reality, vehicle's speed might be lower or higher than the desired speed. Speed limit is generally used when the actual speed data are not available. Recently, transportation professionals have new technologies and instruments to collect traffic data. The use of Global Positioning Systems (GPS) has become a springboard for many transportation related projects. GPS tools can capture, store, and transfer real-time traffic data on the field. Vehicle counts, speed, time, and delay are the key components of information to develop proper signal coordination timing plan. The expanding Geographic Information System (GIS) and GPS technologies have made it easy to collect spatial data (latitude, longitude) with attributes of time and velocity [19-22]. Considering the impact of practical travel speed, Feng [16] has developed a corridor signal optimization method called projection algorithm (PA). It does not introduce the fine-tuning of initial signal timing plan with practical travel speed.
This study proposes an arterial progression optimization approach using link-based bandwidth optimization algorithm and a GPS tool on an arterial. The rest of paper is organized as follows. Section 2 introduces some notations of the bandwidth optimization methodology and provides a link bandwidth optimization algorithm with eight situations to calculate upper/lower interferences and six cases to calculate relative offset between two consecutive intersections. Section 3 presents the new link-based arterial bandwidth optimization algorithm. Section 4 presents a case study to demonstrate the proposed algorithm and compare the performance index of signal timing plans on different speeds, especially the practical vehicles' speed, with simulation models. Finally, Section 5 provides the summary and conclusions.

\section{Link Bandwidth Optimization}

2.1. Notations. $N$ : number of intersections on an arterial; $C$ : signal cycle length; $i$ : intersection; $L_{i, i+1}$ : the link between $i$ and $i+1 ; G_{\mathrm{IBT}, i}$ : inbound through movement green time of $i$; $G_{\mathrm{IBL}, i}$ : inbound left-turn movement green time of $i ; G_{\mathrm{OBT}, i}$ : outbound through movement green time of $i$; $G_{\mathrm{OBL}, i}$ : outbound left-turn movement green time of $i$; $\beta_{i}$ : phase sequence of $i$ for left-turn in the arterial direction, whose value can be $1,2,3$, and 4 , in which (1) "Lead/Lead," meaning IBL, is leading phase and OBL is leading phase, (2) "Lead/Lag," (3) "Lag/Lead," (4) "Lag/Lag"; $O_{i}$ : offset of $i$, start of green time of outbound through movement; $O_{i, i+1}$ : relative offset between $i$ and $i+1 ; \mathrm{RO}_{i, \beta_{i}}$ : relative offset of $G_{\mathrm{IBT}, i}$ with respect to $G_{\mathrm{OBT}, i}$ at $i$ with sequence $\beta_{i}$. Figure 1 shows the calculation of $\mathrm{RO}_{i, \beta_{i}}$.

$T_{i, i+1}$ : travel time from $i$ to $i+1 ; T_{i+1, i}$ : travel time from $i+1$ to $i \operatorname{LI}_{\beta_{i}, \beta_{i+1}}$ : lower interference between $i$ and $i+1$; $\mathrm{UI}_{\beta_{i}, \beta_{i+1}}$ : upper interference between $i$ and $i+1 ; \mathrm{VI}_{\beta_{i}, \beta_{i+1}}$ : valid interference between $i$ and $i+1 ; O s_{i, i+1}$-slack time between $G_{\mathrm{OBT}, i}$ and $G_{\mathrm{OBT}, i+1}$, and $O s_{i, i+1}=G_{\mathrm{OBT}, i}-G_{\mathrm{OBT}, i+1} ; I s_{i, i+1}$ : slack time between $G_{\mathrm{IBT}, i}$ and $G_{\mathrm{IBT}, i+1}$, and $I s_{i, i+1}=G_{\mathrm{IBT}, i}-$ $G_{\mathrm{IBT}, i+1} ; B^{i}$ : link bandwidth of $L_{i, i+1} ; B_{O}^{i}$ : outbound link bandwidth of $L_{i, i+1} ; B_{I}^{i}$ : inbound link bandwidth of $L_{i, i+1}$; $V_{\mathrm{OBT}, i}$ : traffic volume of outbound through movement at $i$; $V_{\mathrm{IBT}, i}$ : traffic volume of inbound through movement at $i$.

2.2. Calculation of Upper/Lower Interference. The bandwidth optimization algorithm developed by Brooks and Little establishes the primary principles of bandwidth optimization. The algorithm was originally developed for two-phase signals. Messer et al. [7] enhanced the original algorithm to handle multiphase signals with left-turn phases, which formed the core of PASSER II [8]. One major limitation of the algorithm is to get the optimal progression bandwidth for an arterial with high number of signalized intersections (e.g., >10). Messer's paper did not include how to calculate relative offsets and did not consider the relative locations of intersections. This section provides detailed descriptions on all possible situations to calculate upper/lower interferences and offset for two consecutive intersections.

Figure 2 illustrates the basic concepts of bandwidth optimization algorithm for three signals with left-turn phases. The maximum progression bandwidth is the sum of outbound bandwidth and inbound bandwidth. The value of 


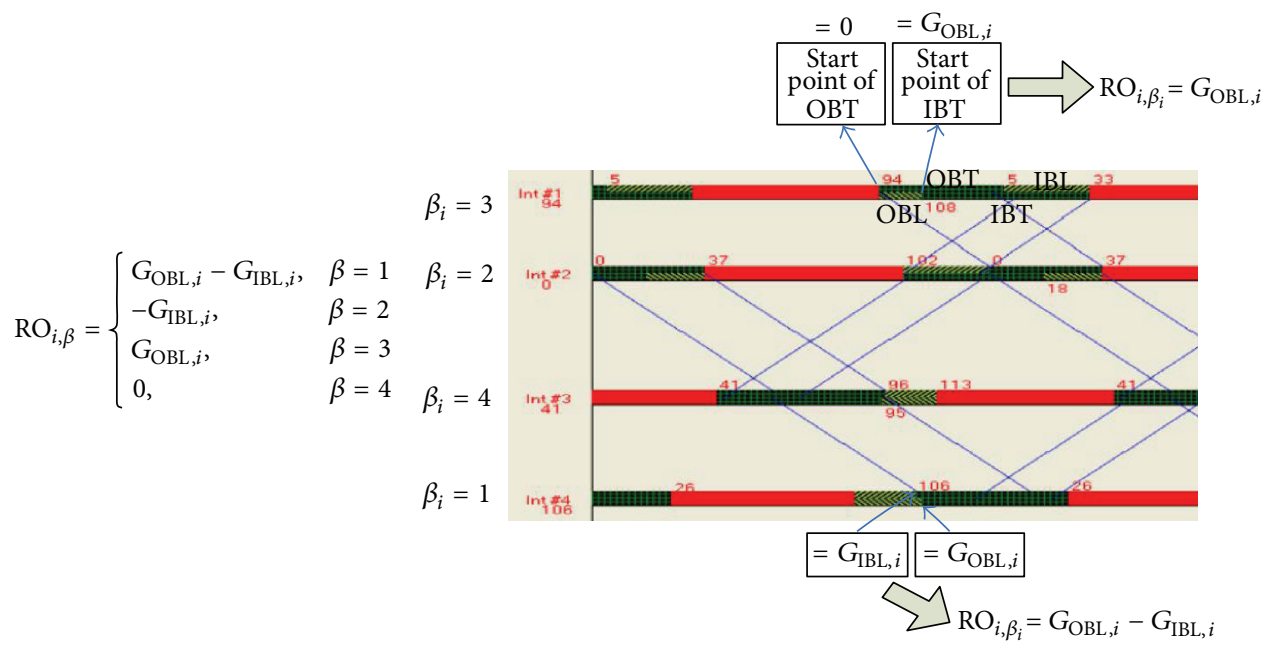

FIGURE 1: Relative offset of $G_{\mathrm{IBT}, i}$ with respect to $G_{\mathrm{OBT}, i}$.

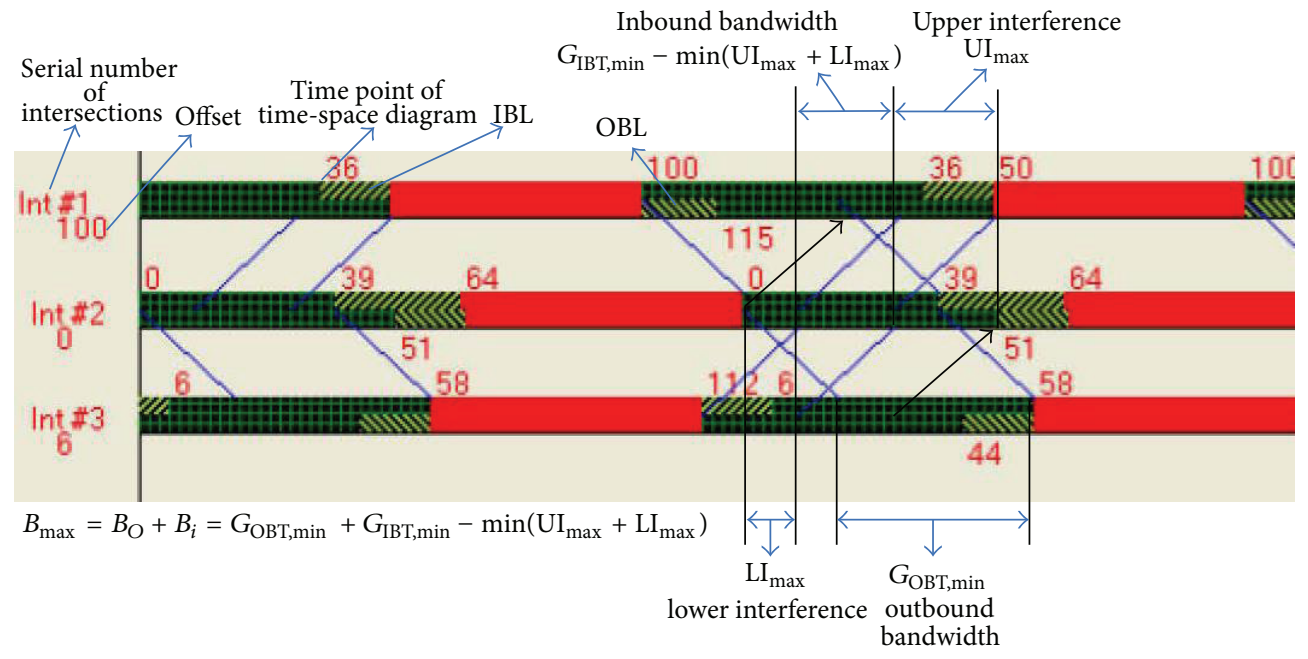

FIGURE 2: Illustration of bandwidth optimization algorithm.

outbound bandwidth is a constant, which equals the minimum outbound through green time $G_{\mathrm{OBT} \text { min }}$. And the inbound bandwidth is determined by minimum total valid interference from other intersections. In Figure 2, the second intersection is the master intersection, which has minimum inbound through green time $G_{\mathrm{IBT} \text {,min }}$. A valid upper interference is by the first intersection, $\mathrm{UI}_{\max }$, and a valid lower interference is by the third intersection, $\mathrm{LI}_{\max }$. Therefore the inbound bandwidth is $G_{I B T, \min }-\min \left(\mathrm{UI}_{\max }+\mathrm{LI}_{\max }\right)$.

The bandwidth optimization principle reveals that the location of $i$ with respect to $i+1$ plays an important role in calculating upper and lower interferences for two consecutive intersections. On the other hand, according to comparison of inbound and outbound through movement green time, there are four situations that ensure correct upper and lower interference calculations.

After calculating upper/lower interferences, there are four additional cases to adjust these upper/lower interferences and obtain the valid interferences, as shown in Table 1. Figure 3 graphically illustrates the calculation of upper interference for the situation 4 . In this situation, the intersection $i$ has the minimum inbound through movement green time, meaning that the master intersection is on the left side of intersection $i+1$.

In Figure 3, phase sequence of the first intersection no. $i$ is "IBL:Lag/OBL:Lead," $\beta_{i}=3$, and phase sequence of the second intersection no. $i+1$ is "IBL:Lead/OBL:Lag," $\beta_{i+1}=$ 2. To calculate upper/lower interference between these two intersections, there are other 15 conditions with different combinations of phase sequences, as shown in Table 2.

2.3. Valid Upper/Lower Interference and Link Bandwidth. All upper/lower interferences to be valid must be less than the minimum inbound through movement green time and greater than the slack time between $G_{\mathrm{IBT}, i}$ and $G_{\mathrm{IBT}, i+1}$, as 


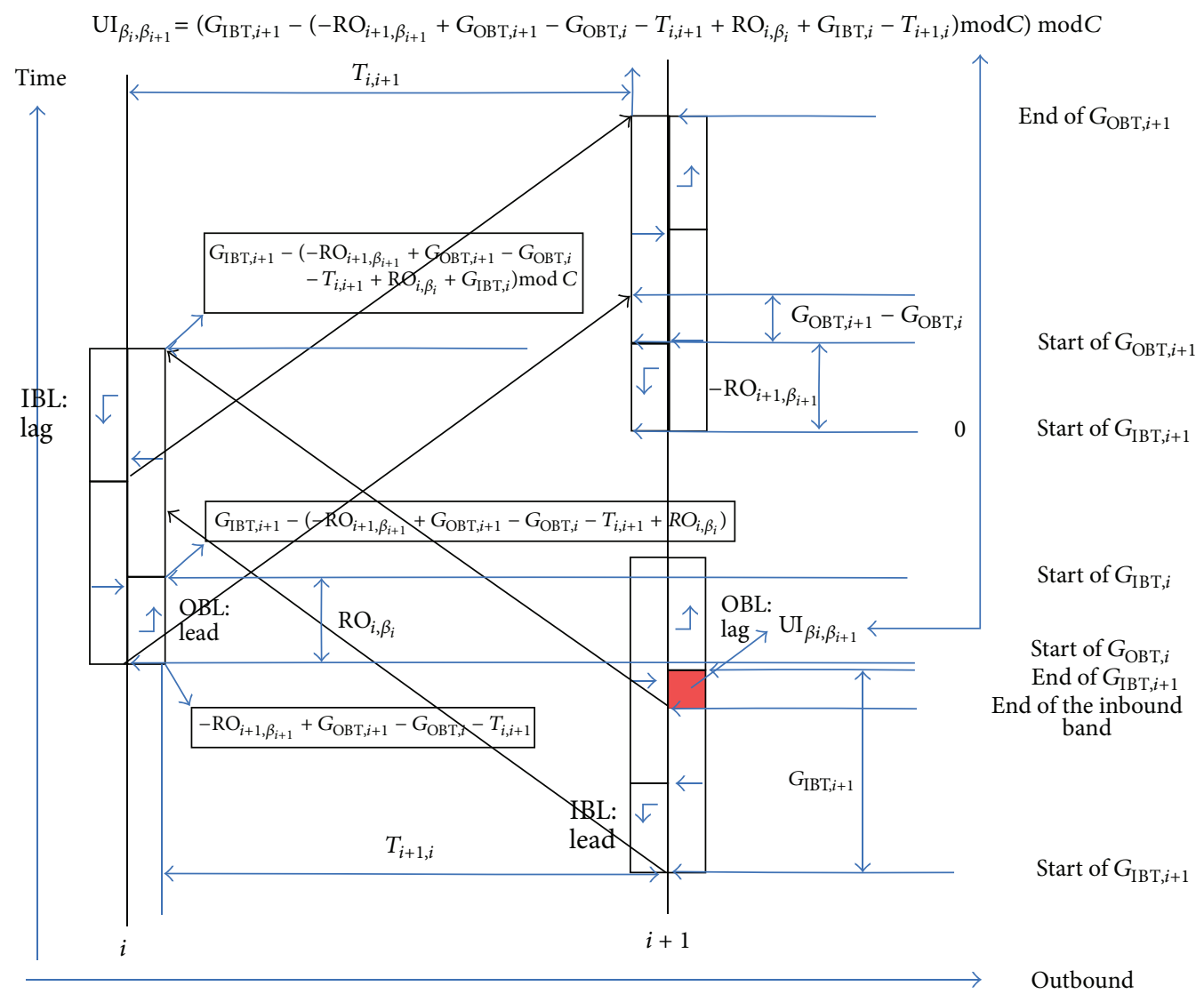

FIGURE 3: The calculation of upper interference for situation 4.

TABLE 1: Calculation of upper/lower interference for link bandwidth.

\begin{tabular}{|c|c|c|}
\hline Common situation & Upper interference & Lower interference \\
\hline$G_{\mathrm{OBT}, i} \leq G_{\mathrm{OBT}, i+1}$ & $\mathrm{UI}_{\beta_{i}, \beta_{i+1}}=G_{I B T, i}-\left(-\mathrm{RO}_{i, \beta_{i}}+T_{i, i+1}\right.$ & $\mathrm{LI}_{\beta_{i}, \beta_{i+1}}=\left(-\mathrm{RO}_{i, \beta_{i}}+T_{i, i+1}-O s_{i+1, i}\right.$ \\
\hline$G_{\mathrm{IBT}, i+1}>G_{\mathrm{IBT}, i}$ & $\left.+\mathrm{RO}_{i+1, \beta_{i+1}}+G_{\mathrm{IBT}, i+1}+T_{i+1, i}\right) \bmod C$ & $\left.+\mathrm{RO}_{i+1, \beta_{i+1}}+T_{i+1, i}\right) \bmod C$ \\
\hline$G_{\mathrm{OBT}, i}>G_{\mathrm{OBT}, i+1}$ & $\mathrm{UI}_{\beta_{i}, \beta_{i+1}}=\left(G_{\mathrm{IBT}, i}-\left(-\mathrm{RO}_{i, \beta_{i}}+G_{\mathrm{OBT}, i}-G_{\mathrm{OBT}, i+1}+T_{i, i+1}\right.\right.$ & $\mathrm{LI}_{\beta_{i}, \beta_{i+1}}=\left(-\mathrm{RO}_{i, \beta_{i}}+T_{i, i+1}-O s_{i+1, i}\right.$ \\
\hline$G_{\mathrm{IBT}, i+1}>G_{\mathrm{IBT}, i+1}$ & $\left.\left.+\mathrm{RO}_{i+1, \beta_{i+1}}+G_{\mathrm{IBT}, i+1}+T_{i+1, i}\right) \bmod C\right) \bmod C$ & $\left.+\mathrm{RO}_{i+1, \beta_{i+1}}+T_{i+1, i}\right) \bmod C$ \\
\hline$G_{\mathrm{OBT}, i+1} \leq G_{\mathrm{OBT}, i}$ & $\mathrm{UI}_{\beta_{i}, \beta_{i+1}}=\left(G_{\mathrm{IBT}, i+1}-\left(-\mathrm{RO}_{i+1, \beta_{i+1}}-T_{i, i+1}\right.\right.$ & $\mathrm{LI}_{\beta_{i}, \beta_{i+1}}=\left(-\mathrm{RO}_{i+1, \beta_{i+1}}-T_{i, i+1}\right.$ \\
\hline$G_{\mathrm{IBT}, i+1} \leq G_{\mathrm{IBT}, i}$ & $\left.\left.+\mathrm{RO}_{i, \beta_{i}}+G_{\mathrm{IBT}, i}-T_{i, i+1}\right) \bmod C\right) \bmod C$ & $\left.-O s_{i, i+1}+\mathrm{RO}_{i, \beta_{i}}-T_{i+1, i}\right) \bmod C$ \\
\hline$G_{\mathrm{OBT}, i+1}>G_{\mathrm{OBT}, i}$ & $\mathrm{UI}_{\beta_{i}, \beta_{i+1}}=\left(G_{\mathrm{IBT}, i+1}-\left(-R O_{i+1, \beta_{i+1}}+G_{\mathrm{OBT}, i+1}-G_{\mathrm{OBT}, i}\right.\right.$ & $\mathrm{LI}_{\beta_{i}, \beta_{i+1}}=\left(-\mathrm{RO}_{i+1, \beta_{i+1}}-T_{i, i+1}\right.$ \\
\hline$G_{\mathrm{OBT}, i+1} \leq G_{\mathrm{OBT}, i}$ & $\left.\left.-T_{i, i+1}+\mathrm{RO}_{i, \beta_{i}}+G_{\mathrm{IBT}, i}-T_{i+1, i}\right) \bmod C\right) \bmod C$ & $\left.-O s_{i, i+1}+\mathrm{RO}_{i, \beta_{i}}-T_{i+1, i}\right) \bmod C$ \\
\hline Special situation & Upper/lower interfence & Interference adjustment \\
\hline$I s_{i, i+1}<0$ & $C+I s_{i, i+1} \leq \mathrm{LI}_{\beta_{i}, \beta_{i+1}} \leq C$ & $\mathrm{LI}_{\beta_{i}, \beta_{i+1}}=\mathrm{LI}_{\beta_{i}, \beta_{i+1}}-C$ \\
\hline $\mathrm{LI}_{\beta_{i}, \beta_{i+1}}<0$ & $C+\mathrm{LI}_{\beta_{i}, \beta_{i+1}} \leq \min \left\{\mathrm{G}_{\mathrm{IBT}, \mathrm{i},}, G_{\mathrm{IBT}, i+1}\right\}$ & $\mathrm{LI}_{\beta_{i}, \beta_{i+1}}=\mathrm{LI}_{\beta_{i}, \beta_{i+1}}+C$ \\
\hline$I s_{i, i+1}<0$ & $C+I s_{i, i+1} \leq \mathrm{UI}_{\beta_{i}, \beta_{i+1}} \leq \mathrm{C}$ & $\mathrm{UI}_{\beta_{i}, \beta_{i+1}}=\mathrm{UI}_{\beta_{i}, \beta_{i+1}}-C$ \\
\hline $\mathrm{UI}_{\beta_{i}, \beta_{i+1}}<0$ & $C+\mathrm{UI}_{\beta_{i}, \beta_{i+1}} \leq \min \left\{G_{\mathrm{IBT}, i}, G_{\mathrm{IBT}, i+1}\right\}$ & $\mathrm{UI}_{\beta_{i}, \beta_{i+1}}=\mathrm{UI}_{\beta_{i}, \beta_{i+1}}+C$ \\
\hline
\end{tabular}

shown in

$$
\begin{aligned}
& I s_{i} \leq \mathrm{LI}_{\beta_{i}, \beta_{i+1}} \leq \min \left\{G_{\mathrm{IBT}, i}, G_{\mathrm{IBT}, i+1}\right\}, \\
& I s_{i} \leq \mathrm{UI}_{\beta_{i}, \beta_{i+1}} \leq \min \left\{G_{\mathrm{IBT}, i}, G_{\mathrm{IBT}, i+1}\right\} .
\end{aligned}
$$

Additionally, because the master intersection has the minimum green time, only one interference (cannot be both) could occur for one combination phase sequence at two intersections, as shown in

$$
\mathrm{VI}_{\beta_{i}, \beta_{i+1}}=\left\{\mathrm{UI}_{\beta_{i}, \beta_{i+1}}, \mathrm{LI}_{\beta_{i}, \beta_{i+1}}\right\} \text {. }
$$


TABLE 2: Phase sequence combinations of two consecutive intersections.

\begin{tabular}{|c|c|c|c|c|c|c|}
\hline \multirow{2}{*}{ Phase sequence combination } & \multicolumn{3}{|c|}{ No. $i$ intersection } & \multicolumn{3}{|c|}{ No. $i+1$ intersection } \\
\hline & IBL & OBL & $\beta$ & IBL & OBL & $\beta$ \\
\hline 1 & \multirow{4}{*}{ Lead } & \multirow{4}{*}{ Lead } & \multirow{4}{*}{$\beta_{i}=1$} & Lead & Lead & $\beta_{i+1}=1$ \\
\hline 2 & & & & Lead & Lag & $\beta_{i+1}=2$ \\
\hline 3 & & & & Lag & Lead & $\beta_{i+1}=3$ \\
\hline 4 & & & & Lag & Lag & $\beta_{i+1}=4$ \\
\hline 5 & \multirow{4}{*}{ Lead } & \multirow{4}{*}{ Lag } & \multirow{4}{*}{$\beta_{i}=2$} & Lead & Lead & $\beta_{i+1}=1$ \\
\hline 6 & & & & Lead & Lag & $\beta_{i+1}=2$ \\
\hline 7 & & & & Lag & Lead & $\beta_{i+1}=3$ \\
\hline 8 & & & & Lag & Lag & $\beta_{i+1}=4$ \\
\hline 9 & \multirow{4}{*}{ Lag } & \multirow{4}{*}{ Lead } & \multirow{4}{*}{$\beta_{i}=3$} & Lead & Lead & $\beta_{i+1}=1$ \\
\hline 10 (Figure 2) & & & & Lead & Lag & $\beta_{i+1}=2$ \\
\hline 11 & & & & Lag & Lead & $\beta_{i+1}=3$ \\
\hline 12 & & & & Lag & Lag & $\beta_{i+1}=4$ \\
\hline 13 & \multirow{4}{*}{ Lag } & \multirow{4}{*}{ Lag } & \multirow{4}{*}{$\beta_{i}=4$} & Lead & Lead & $\beta_{i+1}=1$ \\
\hline 14 & & & & Lead & Lag & $\beta_{i+1}=2$ \\
\hline 15 & & & & Lag & Lead & $\beta_{i+1}=3$ \\
\hline 16 & & & & Lag & Lag & $\beta_{i+1}=4$ \\
\hline
\end{tabular}

Then, a link bandwidth can be gained using

$$
\begin{aligned}
B^{i} & =B_{\mathrm{O}}^{i}+B_{I}^{i} \\
& =\min \left\{G_{\mathrm{OBT}, i}, G_{\mathrm{OBT}, i+1}\right\}+\min \left\{G_{\mathrm{IBT}, i}, G_{\mathrm{IBT}, i+1}\right\}-\mathrm{VI}_{\beta_{i}, \beta_{i+1}} .
\end{aligned}
$$

2.4. Calculation of Relative Offset. Assume that the referencing phase is the start of green time of outbound through movement, there are six cases to calculate the relative offset of two consecutive intersections.

Case 1. Consider $G_{\mathrm{OBT}, i} \leq G_{\mathrm{OBT}, i+1}, G_{\mathrm{IBT}, i+1}>G_{\mathrm{IBT}, i}$, and $\mathrm{VI}_{\beta_{i}, \beta_{i+1}}=\mathrm{UI}_{\beta_{i}, \beta_{i+1}}$, as shown in Figure 4(a).

Then

$$
O_{i}=0, \quad O_{i+1}=T_{i, i+1} \bmod C .
$$

Case 2. Consider $G_{\mathrm{OBT}, i}>G_{\mathrm{OBT}, i+1}$ and $G_{\mathrm{IBT}, i+1}>G_{\mathrm{IBT}, i}$.

Then

$$
O_{i}=0, \quad O_{i+1}=\left(T_{i, i+1}+G_{\mathrm{OBT}, i}-G_{\mathrm{OBT}, i+1}\right) \bmod C .
$$

Case 3. Consider $G_{\mathrm{OBT}, i} \leq G_{\mathrm{OBT}, i+1}, G_{\mathrm{IBT}, i+1}>G_{\mathrm{IBT}, i}$, and $\mathrm{VI}_{\beta_{i}, \beta_{i+1}}=\mathrm{LI}_{\beta_{i}, \beta_{i+1}}$, as shown in Figure 4(b).

$$
\text { Then }
$$

$$
\begin{gathered}
O_{i}=0, \\
O_{i+1}=\left(T_{i, i+1}+G_{\mathrm{OBT}, i}-G_{\mathrm{OBT}, i+1}+K * C\right) \bmod C,
\end{gathered}
$$

where $K$ is an integer. If $T_{i, i+1}+G_{\mathrm{OBT}, i}-G_{\mathrm{OBT}, i+1}<0$, then $K=1$ or else $K=0$.

Case 4. Consider $G_{\mathrm{OBT}, i+1} \leq G_{\mathrm{OBT}, i}, G_{\mathrm{IBT}, i+1} \leq G_{\mathrm{IBT}, i}$, and $\mathrm{VI}_{\beta_{i}, \beta_{i+1}}=\mathrm{UI}_{\beta_{i}, \beta_{i+1}}$.
Then

$$
O_{i}=K * C-T_{i, i+1}, \quad O_{i+1}=0 .
$$

Case 5. Consider $G_{\mathrm{OBT}, i+1}>G_{\mathrm{OBT}, i}$ and $G_{\mathrm{IBT}, i+1} \leq G_{\mathrm{IBT}, i}$. Then

$$
\mathrm{O}_{i}=K * C-T_{i, i+1}+G_{\mathrm{OBT}, i+1}-G_{\mathrm{OBT}, i}, \quad O_{i+1}=0 .
$$

Case 6. Consider $G_{\mathrm{OBT}, i+1} \leq G_{\mathrm{OBT}, i}, G_{\mathrm{IBT}, i+1} \leq G_{\mathrm{IBT}, i}$, and $\mathrm{VI}_{\beta_{i}, \beta_{i+1}}=\mathrm{LI}_{\beta_{i}, \beta_{i+1}}$.

Then

$$
O_{i}=K * C-T_{i, i+1}+G_{\mathrm{OBT}, i+1}-G_{\mathrm{OBT}, i}, \quad O_{i+1}=0 .
$$

2.5. Example of Link Bandwidth Optimization Algorithm. The signal timing plans of two intersections are shown below. The distance between them is $2015 \mathrm{ft}$, and the speed limit is $40 \mathrm{mph}$; then travel time is $34 \mathrm{~s}$ and cycle length is $130 \mathrm{~s}$ :

$$
\begin{aligned}
G_{\mathrm{IBT}, i} & =51, & & G_{\mathrm{OBT}, i}=49, \\
G_{\mathrm{IBL}, i} & =20, & & G_{\mathrm{OBL}, i}=18, \\
G_{\mathrm{IBT}, i+1} & =45, & & G_{\mathrm{OBT}, i+1}=36, \\
G_{\mathrm{IBL}, i+1} & =29, & & G_{\mathrm{OBL}, i+1}=20 .
\end{aligned}
$$

Then, $G_{\mathrm{OBT}, i+1} \leq G_{\mathrm{OBT}, i}$ and $G_{\mathrm{IBT}, i+1} \leq G_{\mathrm{IBT}, i}$ are fit for common situation 3 in Table 1 . Table 3 shows the results of upper/lower interference calculation, including 16 phase sequence combinations.

According to the rule of valid interference in Section 2.3 and calculation of offset in Section 2.4, we can get the valid interference, link bandwidth, and offset relative to phase sequences, as shown in Table 3 (bold text), including 10 phase sequence combinations (not all 16 combinations have valid interference). 
TABLE 3: Upper/lower interferences of two consecutive intersections.

\begin{tabular}{|c|c|c|c|c|c|c|c|c|}
\hline \multicolumn{9}{|l|}{ Phase sequence } \\
\hline Intersection $i+1$ & 1 & 1 & 1 & 1 & 2 & 2 & 2 & 2 \\
\hline Intersection $i$ & 1 & 2 & 3 & 4 & 1 & 2 & 3 & 4 \\
\hline \multicolumn{9}{|c|}{$\begin{array}{l}\text { Interference of intersection } \\
i+1 \text { from intersection } i\end{array}$} \\
\hline Upper interference & 55 & $\begin{array}{c}\text { Common: } \\
45-(-(20-29)-34+(-20)+51-34)=73\end{array}$ & 35 & 53 & 35 & 53 & 15 & 33 \\
\hline Lower interference & -74 & $\begin{array}{c}\text { Common: } \\
-(-9)-34-(49-36)+(-20)-34=-92 \\
\text { Special: }-\mathbf{9 2}+\mathbf{1 3 0}=\mathbf{3 8}<\mathbf{4 5} \\
\text { valid interference }\end{array}$ & -54 & -72 & -54 & -72 & -34 & -52 \\
\hline Link bandwidth & - & $45+36-38=43$ & $45+36-35=46$ & - & 46 & - & 66 & 48 \\
\hline Offset of intersection $i$ & - & $130-34+36-49=83$ & $130-34=96$ & - & 96 & - & 96 & 96 \\
\hline \multicolumn{9}{|l|}{ Phase sequence } \\
\hline Intersection $i+1$ & 3 & 3 & 3 & 3 & 4 & 4 & 4 & 4 \\
\hline Intersection $i$ & 1 & 2 & 3 & 4 & 1 & 2 & 3 & 4 \\
\hline \multicolumn{9}{|l|}{ Interference } \\
\hline Upper interference & 84 & 102 & 64 & 82 & 64 & 82 & 44 & 62 \\
\hline Lower interference & 27 & 9 & -83 & 29 & -83 & 29 & -63 & -81 \\
\hline Link bandwidth & 54 & 72 & - & 52 & - & 52 & 37 & - \\
\hline Offset of intersection $i$ & 83 & 83 & - & 83 & - & 83 & 96 & - \\
\hline
\end{tabular}

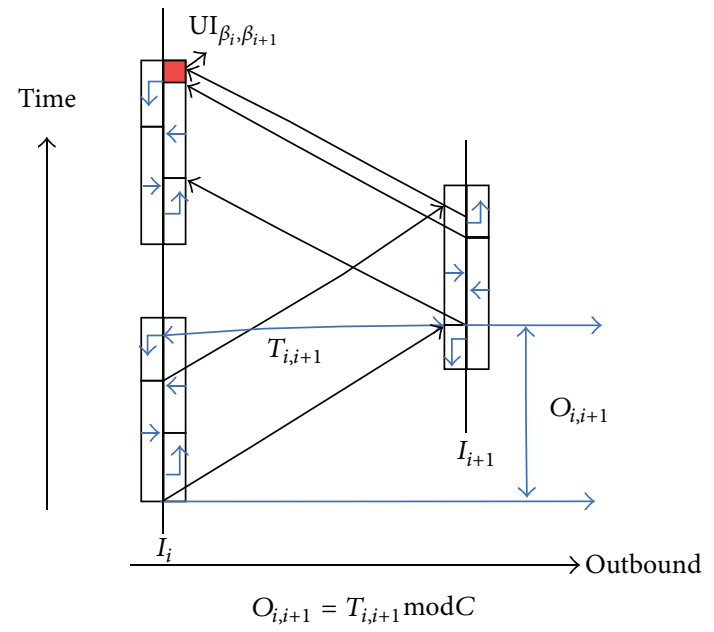

(a) Case 1: upper interference

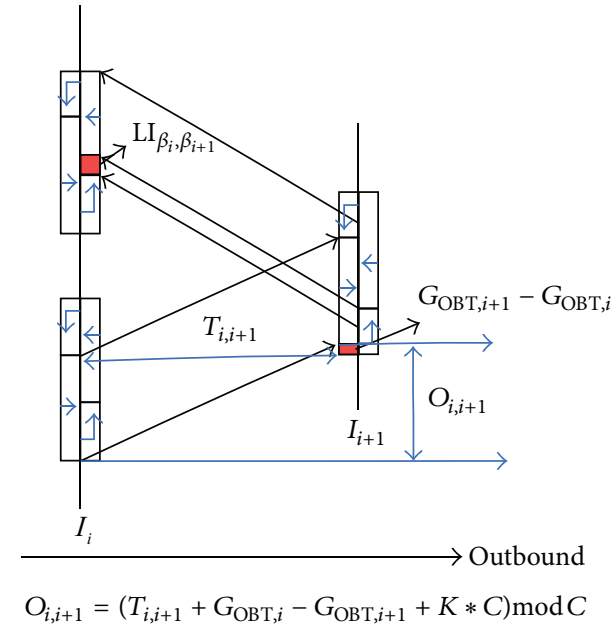

(b) Case 3: lower interference

FIGURE 4: The calculation of relative offset.

\section{Link-Based Arterial Bandwidth Optimization Algorithm}

3.1. Measures of Effectiveness (MOE). Bandwidth efficiency and attainability are two measures to describe the quality of a coordinated signal timing plan. Both are computed from a time-space diagram that represents the signal timing plan.
The two parameters are first defined by Messer et al. [7, 8], as shown in

$$
\begin{gathered}
E_{B}=\frac{\left(B_{\mathrm{out}}+B_{\mathrm{in}}\right)}{2 C} \times 100 \%, \\
A_{c}=\frac{B_{\mathrm{out}}+B_{\text {in }}}{G_{\mathrm{OBT}, \text { min }}+G_{\mathrm{IBT}, \text { min }}} \times 100 \%,
\end{gathered}
$$




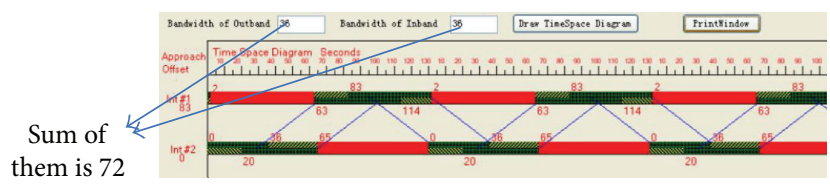

(a) Maximum link bandwidth

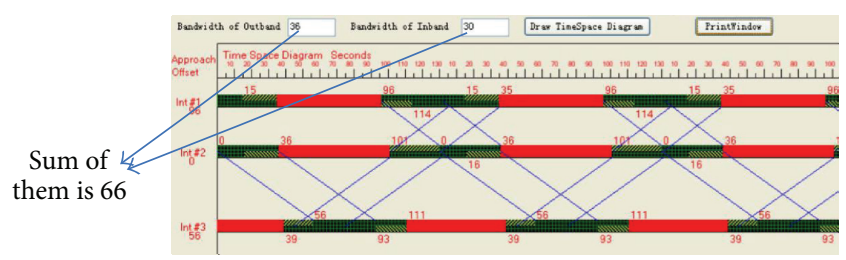

(b) Possible link and arterial bandwidth

FIGURE 5: Example of maximum and possible link bandwidth and arterial bandwidth.

where $E_{B}$ arterial bandwidth efficiency, $B_{\text {out }}$ arterial bandwidth of outbound, $B_{\text {in }}$ arterial bandwidth of inbound, and $A_{c}$ the attainability for a given cycle length.

Besides the arterial bandwidth efficiency and attainability, maximum link bandwidth, possible link bandwidth, and link bandwidth attainability are first proposed as additional measures of effectiveness in our research. Maximum link bandwidth is the maximum value of a link bandwidth obtained using link bandwidth optimization algorithm. Possible link bandwidth is the value of a link bandwidth when the signal system has the optimal arterial progression bandwidth. In this case, the link bandwidth may not be the best solution to get the arterial bandwidth. In Figure 5, the maximum link bandwidth between the first and the second intersections is 72 seconds, while the possible link bandwidth is only 66 seconds to obtain the optimal arterial bandwidth (offset and phase sequence have been changed).

$B_{\max }^{i}$ is maximum link bandwidth of $L_{i}, B_{i}$ possible link bandwidth of $L_{i}$, and $\alpha_{i}$ link bandwidth attainability, defined as ratio of $B_{i}$ over $B_{\max }^{i}$, shown in

$$
\alpha_{i}=\frac{B_{i}}{B_{\max }^{i}} .
$$

3.2. Calculation of Link Bandwidth. After the outbound direction is determined, link bandwidth $B^{i}$ with phase sequence $\beta_{i}, \beta_{i+1}$, and offset $O_{i, i+1}$ can be calculated. The optimized results of $L_{i, i+1}$ are shown in Table 4 and are sorted in a descending order by $B^{i}$. Both $\beta_{i}$ and $\beta_{i+1}$ can be $1,2,3$, and 4 ; therefore the total number of possible solutions is 16 . It is noted that not all 16 solutions yield a valid upper or lower interference. Only 9 valid solutions are included in Table 4, using two intersecions on Kietzke Lane, Reno, NV, USA.

Similarly, the link bandwidth $B^{i+1}$ with phase sequence $\beta_{i+1}^{\prime}$ (intermediate variable to differentiate it from $\left.\beta_{i+1}\right), \beta_{i+2}$ and offset $O_{i+1, i+2}$ can be calculated. The optimized results of $L_{i+1, i+2}$ are shown in Table 5 and sorted in a descending order by $B^{i+1}$.

Using the same approach, the link bandwidth $B^{i+2}$ with phase sequence $\beta_{i+2}^{\prime}, \beta_{i+3}$ and offset $O_{i+2, i+3}$ can be calculated. The optimized results of $L_{i+2, i+3}$ are shown in Table 6 and sorted in a descending order by $B^{i+2}$.

3.3. Selection of Phase Sequence. Using the conditions $\beta_{i+1}=$ $\beta_{i+1}^{\prime}$ and $\beta_{i+2}=\beta_{i+2}^{\prime}$, one can get many solutions of phase sequences for the intersections on this arterial. Importance of each link bandwidth may be different from one another on an arterial. There are many factors that impact the value of the importance, such as traffic volumes (through movement, leftturn), road configuration, and passing pedestrians. If traffic volume of through movement from $i$ and $i+1$ is more than others, then link bandwidth of $L_{i, i+1}$ is more important than others. $\omega_{i}$ is defined as a weighted factor that reflects the importance of link bandwidth attainability. $E_{m}$ is defined as the efficiency for solution $m$ of a coordinated signal timing plan

$$
\begin{gathered}
E_{m}=\sum_{i}^{N} \omega_{i} \alpha_{i}, \\
\omega_{i}=\frac{\left(V_{\mathrm{OBT}, i}+V_{\mathrm{IBT}, i+1}\right)}{\sum_{i}\left(V_{\mathrm{OBT}, i}+V_{\mathrm{IBT}, i+1}\right)} .
\end{gathered}
$$

The best solution is the one that has the maximum efficiency $E_{m}$. In this example,

$$
\begin{aligned}
\omega_{1}= & \frac{\left(V_{\mathrm{OBT}, i}+V_{\mathrm{IBT}, i+1}\right)}{\sum_{i}\left(V_{\mathrm{OBT}, i}+V_{\mathrm{IBT}, i+1}\right)} \\
= & \frac{(820+492)}{(820+492)+(903+625)+(971+6483)} \\
= & 0.3055, \\
& \omega_{2}=0.3559, \quad \omega_{3}=0.3386 .
\end{aligned}
$$

Table 7 shows five solutions of different phase sequences, based on the data in Tables 4, 5, and 6 . The weighted factor of each link is calculated using through traffic volumes. As can be seen, solution 2 is the best solution, which has an efficiency of 0.9755 . The number of solutions is dependent on the optimized result. Table 7 just shows five of the solutions.

3.4. Calculation of Offset. The inbound through movement green time determines which intersection is the reference intersection for one link and how the relative offset of each intersection is calculated. According to the value of inbound through movement green time, there are four cases to calculate the offsets of $i, i+1$, and $i+2$.

Case 1. Consider $G_{\mathrm{IBT}, i+1}>G_{\mathrm{IBT}, i}$ and $G_{\mathrm{IBT}, i+2}>G_{\mathrm{IBT}, i+1}$; then

$$
O_{i}=0, \quad O_{i+1}=O_{i, i+1}, \quad O_{i+2}=O_{i, i+1}+O_{i+1, i+2} .
$$


TABLE 4: Optimized results for $L_{i, i+1}$ on Kietzke Lane $\left(G_{\mathrm{IBT}, i+1}<G_{\mathrm{IBT}, i}\right.$, 2nd Steet and Mill Street).

\begin{tabular}{lccccccccc}
\hline Timing parameter & & & \multicolumn{5}{c}{ Valid solutions } \\
& 1 & 2 & 3 & 4 & 5 & 6 & 7 & 3 \\
\hline$\beta_{i}$ & 2 & 3 & 1 & 4 & 2 & 4 & 1 & 2 \\
$\beta_{i+1}$ & 3 & 2 & 3 & 3 & 4 & 2 & 1 & 2 \\
$B^{i}$ & 72 & 66 & 54 & 52 & 52 & 48 & 46 & 46 & 43 \\
$\alpha_{i}$ & 1.0 & 0.92 & 0.75 & 0.72 & 0.72 & 0.67 & 0.64 & 0.64 & 0.60 \\
$O_{i, i+1}$ & 83 & 96 & 83 & 83 & 83 & 96 & 96 & 96 \\
\hline
\end{tabular}

TABLE 5: Optimized results for $L_{i+1, i+2}$ on Kietzke Lane $\left(G_{\mathrm{IBT}, i+2}>G_{\mathrm{IBT}, i+1}\right.$, Mill Street and Vassar Street).

\begin{tabular}{|c|c|c|c|c|c|c|c|c|c|c|c|c|c|c|c|}
\hline \multirow{2}{*}{ Timing parameter } & \multicolumn{15}{|c|}{ Valid solutions } \\
\hline & 1 & 2 & 3 & 4 & 5 & 6 & 7 & 8 & 9 & 10 & 11 & 12 & 13 & 14 & 15 \\
\hline$\overline{\beta_{i+1}^{\prime}}$ & 1 & 1 & 1 & 2 & 2 & 2 & 4 & 4 & 4 & 2 & 3 & 1 & 4 & 3 & 3 \\
\hline$\beta_{i+2}$ & 1 & 3 & 4 & 1 & 2 & 4 & 3 & 1 & 4 & 3 & 3 & 2 & 2 & 1 & 4 \\
\hline$B^{i+1}$ & 81 & 81 & 81 & 81 & 81 & 81 & 81 & 73 & 72 & 71 & 70 & 64 & 55 & 53 & 52 \\
\hline$\alpha_{i+1}$ & 1.0 & 1.0 & 1.0 & 1.0 & 1.0 & 1.0 & 1.0 & 0.90 & 0.89 & 0.88 & 0.86 & 0.79 & 0.68 & 0.65 & 0.64 \\
\hline $\mathrm{O}_{i+1, i+2}$ & 56 & 56 & 56 & 37 & 56 & 37 & 56 & 56 & 56 & 37 & 56 & 56 & 56 & 56 & 56 \\
\hline
\end{tabular}

TABLE 6: Optimized results for $L_{i+2, i+3}$ on Kietzke Lane $\left(G_{\mathrm{IBT}, i+3}<G_{\mathrm{IBT}, i+2}\right.$, Vassar Street and Plumb Lane).

\begin{tabular}{|c|c|c|c|c|c|c|c|c|c|c|c|c|}
\hline \multirow{2}{*}{ Timing parameter } & \multicolumn{12}{|c|}{ Valid solutions } \\
\hline & 1 & 2 & 3 & 4 & 5 & 6 & 7 & 8 & 9 & 10 & 11 & 12 \\
\hline$\overline{\beta_{i+2}^{\prime}}$ & 2 & 4 & 1 & 2 & 2 & 3 & 4 & 1 & 4 & 1 & 2 & 3 \\
\hline$\beta_{i+3}$ & 3 & 3 & 3 & 1 & 4 & 3 & 1 & 1 & 4 & 4 & 2 & 2 \\
\hline$B^{i+2}$ & 98 & 83 & 82 & 80 & 78 & 65 & 63 & 62 & 61 & 60 & 58 & 54 \\
\hline$\alpha_{i+2}$ & 1.0 & 0.85 & 0.84 & 0.82 & 0.80 & 0.63 & 0.64 & 0.63 & 0.62 & 0.61 & 0.59 & 0.55 \\
\hline$O_{i+2, i+3}$ & 81 & 81 & 81 & 81 & 81 & 81 & 81 & 81 & 81 & 81 & 81 & 86 \\
\hline
\end{tabular}

Case 2. Consider $G_{\mathrm{IBT}, i+1}>G_{\mathrm{IBT}, i}$ and $G_{\mathrm{IBT}, i+2} \leq G_{\mathrm{IBT}, i+1}$; then

$$
\begin{gathered}
O_{i}=0, \quad O_{i+1}=O_{i, i+1}, \\
O_{i+2}=O_{i, i+1}-O_{i+1, i+2}+K * C
\end{gathered}
$$

where $K$ is an integer. If $O_{i, i+1}-O_{i+1, i+2}<0$, then $K=1$ or else $K=0$.

Case 3. Consider $G_{\mathrm{IBT}, i+1} \leq G_{\mathrm{IBT}, i}$ and $G_{\mathrm{IBT}, i+2}>G_{\mathrm{IBT}, i+1}$; then

$$
O_{i}=O_{i, i+1}, \quad O_{i+1}=0, \quad O_{i+2}=O_{i+1, i+2} .
$$

Case 4. Consider $G_{\mathrm{IBT}, i+1} \leq G_{\mathrm{IBT}, i}$ and $G_{\mathrm{IBT}, i+2} \leq G_{\mathrm{IBT}, i+1}$; then

$$
O_{i}=O_{i, i+1}, \quad O_{i+1}=0, \quad O_{i+2}=C-O_{i+1, i+2} .
$$

In Table 7, solution 2 is the best solution of phase sequences on this arterial. Using the algorithm of calculating the above offset, one can get the offset for each intersection.

(1) Consider $G_{\mathrm{IBT}, i+1}<G_{\mathrm{IBT}, i}, G_{\mathrm{IBT}, i+2}>G_{\mathrm{IBT}, i+1}$; then

$$
O_{i}=O_{i, i+1}=96, \quad O_{i+1}=0, \quad O_{i+2}=O_{i+1, i+2}=56 .
$$

(2) Consider $G_{\mathrm{IBT}, i+3}<G_{\mathrm{IBT}, i+2}$; then

$$
\begin{aligned}
O_{i+2}=O_{i+1, i+2} & =56 \\
O_{i+3}=K * C+\left(O_{i+1, i+2}-O_{i+2, i+3}\right) & =130+(56-81)=105 .
\end{aligned}
$$

\section{Case Study}

4.1. Site Description. A Windows program bandwidth optimization and time space diagram (BOTSD) was developed based on the proposed algorithm. Time-space diagrams can be produced by BOTSD with additional information to show the start and end of each phase. This information is useful for field implementation of signal timing plans, providing easiness of verifying that the current plan is running.

Kietzke Lane, an arterial in Reno, NV, USA, is selected as a case study to check the performance of the proposed bandwidth optimization algorithm. There are eight intersections (E 2nd Street no. 1, Mill Street no. 2, Vassar Street no. 3, Plumb Lane no. 4, Grove Street no. 5, Gentry Way no. 6, Moana Lane no. 7, and Peckham Lane no. 8) on the Kietzke Lane selected in this study. The coordinated cycle length of this arterial is 130 seconds (16:00 pm-18:00 pm) and speed limit is $40 \mathrm{mph}$. Table 8 shows lengths of phase split in the initial signal timing plan of Kietzke Lane. This plan is suitable for real traffic volumes of each isolated intersection, 
TABLE 7: Selected solutions of different phase sequences on Kietzke Lane (2nd Street $\rightarrow$ Plumb Lane).

\begin{tabular}{|c|c|c|c|c|c|c|c|c|c|c|}
\hline$m$ & & & & & & & & & & \\
\hline$I_{i}$ & $\beta_{i}$ & $\alpha_{i}$ & $\beta_{i}$ & $\alpha_{i}$ & $\beta_{i}$ & $\alpha_{i}$ & $\beta_{i}$ & $\alpha_{i}$ & $\beta_{i}$ & $\alpha_{i}$ \\
\hline 1 & 2 & 1 & 3 & 0.92 & 3 & 0.92 & 3 & 0.92 & 1 & 0.75 \\
\hline 2 & 3 & 0.86 & 2 & 1 & 2 & 1 & 2 & 1 & 3 & 0.86 \\
\hline 3 & 3 & 0.63 & 2 & 1 & 4 & 0.85 & 1 & 0.84 & 3 & 0.63 \\
\hline 4 & 3 & - & 3 & - & 3 & - & 3 & - & 3 & - \\
\hline$E_{m}$ & - & 0.8249 & - & 0.9755 & - & 0.9247 & - & 0.9214 & - & 0.7485 \\
\hline
\end{tabular}

TABLE 8: Phase splits in the initial signal timing plan of Kietzke Lane (2nd Street $\rightarrow$ Peckham Lane).

\begin{tabular}{|c|c|c|c|c|c|c|c|c|c|}
\hline INTID & SBL & NBT & WBL & EBT & NBL & SBT & EBL & WBT & Distance $(\mathrm{ft})$ \\
\hline 1 & 18 & 51 & 20 & 41 & 20 & 49 & 30 & 31 & \\
\hline 2 & 20 & 45 & 25 & 40 & 29 & 36 & 25 & 40 & 2015 \\
\hline 3 & 18 & 54 & 23 & 35 & 17 & 55 & 18 & 40 & 3294 \\
\hline 4 & 22 & 48 & 18 & 42 & 20 & 50 & 22 & 38 & 2600 \\
\hline 5 & 16 & 79 & - & 35 & 16 & 79 & - & 35 & 1841 \\
\hline 6 & 15 & 80 & - & 35 & 18 & 77 & - & - & 2180 \\
\hline 7 & 22 & 40 & 20 & 48 & 18 & 44 & 19 & 49 & 724 \\
\hline 8 & 36 & 41 & 21 & 32 & 16 & 61 & 19 & 34 & 2168 \\
\hline
\end{tabular}

TABLE 9: Traffic volumes of every intersection in the Kietzke Lane from field data.

\begin{tabular}{lcccccccccccc}
\hline INTID & NBL & NBT & NBR & SBL & SBT & SBR & EBL & EBT & EBR & WBL & WBT & WBR \\
\hline 1 & $\mathbf{2 2 6}$ & 820 & 169 & $\mathbf{5 6}$ & 430 & 54 & 248 & 585 & 143 & 160 & 418 & 63 \\
2 & $\mathbf{2 2 0}$ & 903 & 259 & $\mathbf{1 2 0}$ & 492 & 121 & 166 & 684 & 134 & 318 & 742 & 146 \\
3 & $\mathbf{9 6}$ & 971 & 120 & $\mathbf{1 0 9}$ & 625 & 146 & 149 & 238 & 100 & 200 & 408 & 256 \\
4 & $\mathbf{2 5 3}$ & 780 & 313 & $\mathbf{2 9 9}$ & 483 & 143 & 230 & 870 & 244 & 282 & 757 & 168 \\
5 & $\mathbf{8 9}$ & 1193 & 72 & $\mathbf{6}$ & 800 & 148 & 113 & 71 & 70 & 128 & 98 & 30 \\
6 & $\mathbf{1 1 9}$ & 1143 & 51 & $\mathbf{3 0}$ & 883 & 79 & 170 & 56 & 170 & 61 & 66 \\
7 & $\mathbf{1 6 6}$ & 897 & 168 & $\mathbf{2 9 6}$ & 738 & 140 & 250 & 631 & 111 & 257 & 681 & 176 \\
8 & $\mathbf{7 1}$ & 845 & 249 & $\mathbf{3 0 5}$ & 633 & 168 & 81 & 419 & 19 & - & 514 & 300 \\
Total & $\mathbf{1 2 4 0}$ & 7552 & 1401 & $\mathbf{1 2 2 1}$ & 5084 & 999 & 1407 & 3554 & 991 & 1406 & 3684 & 1185 \\
\hline
\end{tabular}

shown in Table 9, not fit for the whole arterial. Left-turn traffic volumes were much lower than through movement traffic volumes, so the optimal arterial progression bandwidth can enable more drivers pass through the arterial systems without stops. In this case study, based on this timing plan, we optimized phase sequences and offsets to obtain best solution of signal timing plan with optimal link bandwidth and arterial bandwidth.

4.2. Optimized Results Using Messer's Algorithm. Figure 6 shows time-space diagram of Kietzke Lane drawn by BOTSD, using Messer's algorithm when southbound is the outbound direction. The outbound arterial bandwidth is 36 seconds and 14 seconds for the inbound. If northbound is selected as the outbound direction, then the second intersection is the master intersection, which has the minimum through movement green time. The outbound arterial bandwidth is 40 seconds and 13 seconds for the inbound. If a vehicle arrives at intersection no. 1 at the beginning of outbound through movement, it will stop and wait at intersection no. 1 and the offset of intersection no. 1 can be adjusted, as shown in Figure 6.

4.3. Optimized Results Using the Proposed Algorithm. Southbound is the outbound direction. Figure 7 shows time-space diagram for this arterial drawn by BOTSD using the new proposed method in southbound. The arterial outbound bandwidth is 30 seconds, and 28 seconds for the inbound. Arterial bandwidth efficiency is $22.31 \%$, and bandwidth attainability is $75.32 \%$, which means a fair progression according to the guidelines of bandwidth efficiency [1].

Using the same method of fine-tuning, we have got time-space diagrams with other travel speeds, which are less than the speed limit and same for every link. In this case study, the speed limit is $40 \mathrm{mph}$; we can use $38 \mathrm{mph}$, $35 \mathrm{mph}$, and $32 \mathrm{mph}$ to design the signal timing plans with 


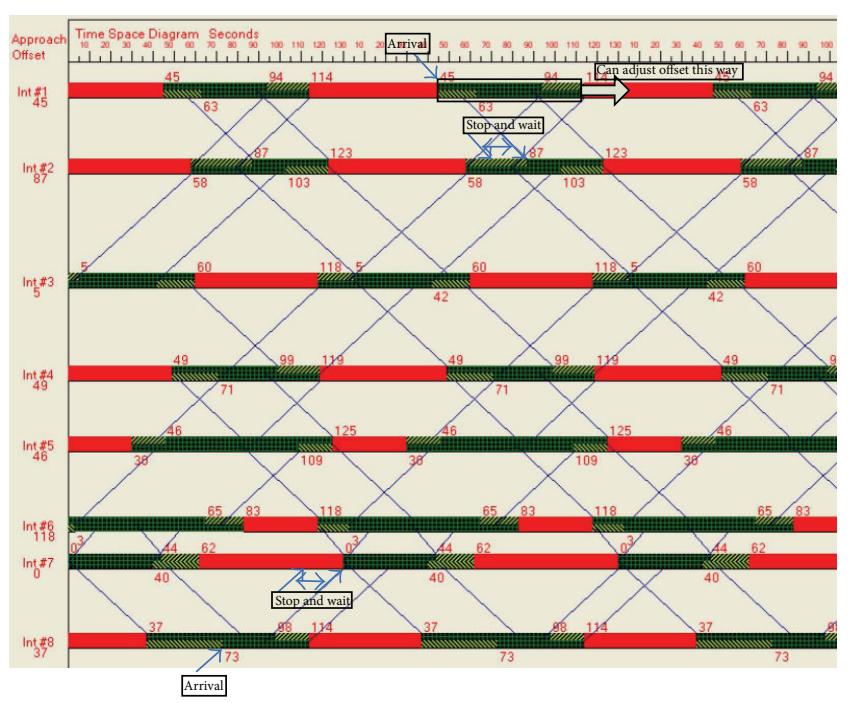

FIGURE 6: TSD of signal timing plan with Messer's algorithm.

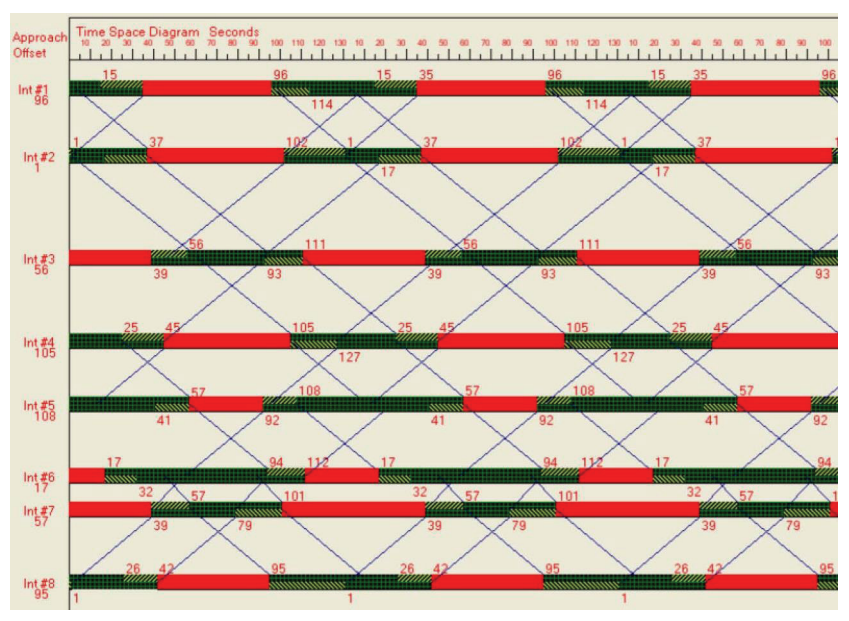

FIGURE 7: TSD of signal timing plan with proposed algorithm.

the proposed bandwidth optimization algorithm and get the TSD with maximum progression of arterial bandwidth and link bandwidth for comparison.

\subsection{Fine-Tuning of Signal Timing Plan with Practical Travel Speed}

4.4.1. Travel Time Data Collection with GPS Tool. We have collected more than ten study runs of GPS data at the PM peak hours, using GPS receiver and GPS2LT [23]. Then, the actual travel time and speed can be obtained by the PC travel for Windows [24]. Based on the results of the actual travel time and speed for links shown in Figure 8, the actual travel speeds for most links are less than speed limit. Additionally, all the standard deviations of travel speed for ten runs are less than $3 \mathrm{mph}$.

4.4.2. Analysis of Initial Signal Timing. Using the GPS and TS tool and the collected GPS data, we have drawn the GPS trajectory on the TSD for three types of study runs for PM peak hours (16:00 pm-18:00 pm). In Figure 9, the arterial road progression bandwidth of southbound is 12 seconds and 29 seconds in northbound. The car can go through all the 8 intersections on the TSD with the speed of $40 \mathrm{mph}$ in the southbound for just two of ten runs. However, on the northbound, the car must stop at least one time no matter when it entered the system.

4.4.3. Signal Timing with Practical Speed. According to the results of fine-tuning of initial signal timing plan link by link, we have got the best solution of TSD for the arterial. The arterial progression bandwidth of southbound is 28 seconds, and 39 seconds in northbound. The bandwidth efficiency is $25.8 \%$. Attainability of every link is more than $85 \%$ and three of them are equal to $100 \%$, as shown in Figure 10.

\subsection{Comparison of Results}

4.5.1. MOE Results. Table 10 shows the link bandwidth attainability and arterial bandwidth efficiency of eight intersections on Kietzke Lane with Messer's algorithm and the proposed algorithm.

According to arterial bandwidth efficiency and attainability in Figures 9 and 10, and link bandwidth attainability in Table 10, the solution of coordinated signal timing plan from the proposed algorithm is much better than that from Messer's algorithm whether the outbound direction is southbound or northbound. If a vehicle arrives at intersection no. 1 at the beginning of outbound through movement, it will stop and wait at intersection no. 1, the offset of intersection no. 1 can be adjusted, as shown in Figure 9. However, the timespace diagrams in Figure 10 have improved these problems.

In the other way, simulation results from SYNCHRO show that the proposed algorithm is better than Messer's algorithm. Total intersection control delay of 8 intersections with optimized signal timing plan with proposed algorithm is $266.2 \mathrm{~s}$, less than that of Messer's algorithm $288 \mathrm{~s}$. Total Link delay in arterial direction with proposed algorithm is $401.3 \mathrm{~s}$, less than that of Messer's algorithm $445.2 \mathrm{~s}$.

Additionally, when the number of signals in a system increases, it becomes more difficult to obtain a good bandwidth solution using Messer's algorithm. In some cases there is no valid solution with arterial bandwidth. However, the new proposed algorithm can be used to obtain an optimal solution of coordinated signal timing plan with both optimal link bandwidth and optimal arterial bandwidth.

4.5.2. Simulation Results Comparison of Speed Limit and Practical Speed. The simulation model of this arterial is built up in VISSIM 5.30. Individual parameters in VISSIM 5.30 were adjusted and tuned with model calibration and validation. To reduce stochastic variation, ten random-seeded runs were conducted in VISSIM 5.30 for the case study. At the same time, the signal timing plan has been input into SYNCHRO 7.0 to get the control delay for the intersections and links on the arterial and check the traffic flow lines on the TSD. Average speed and delay per vehicle for the whole network, 


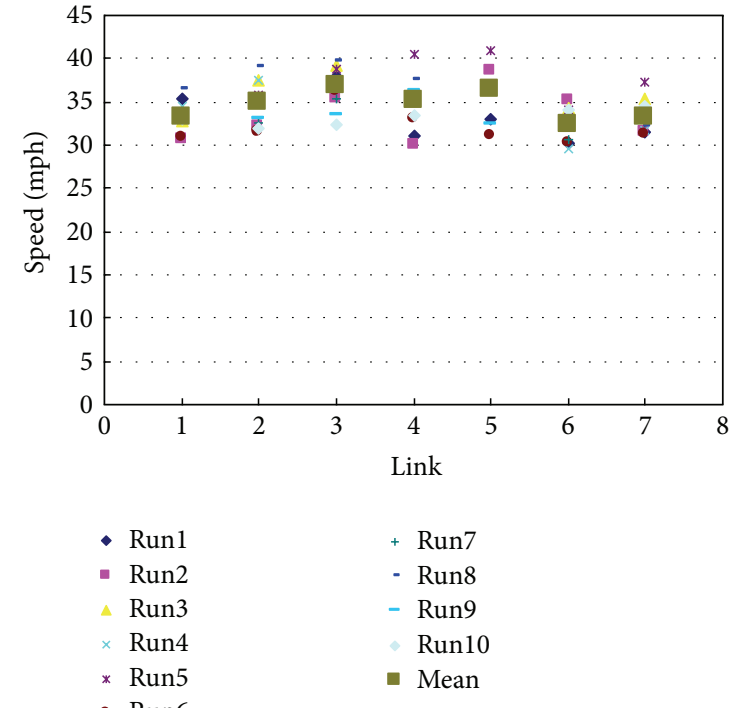

(a) Southbound

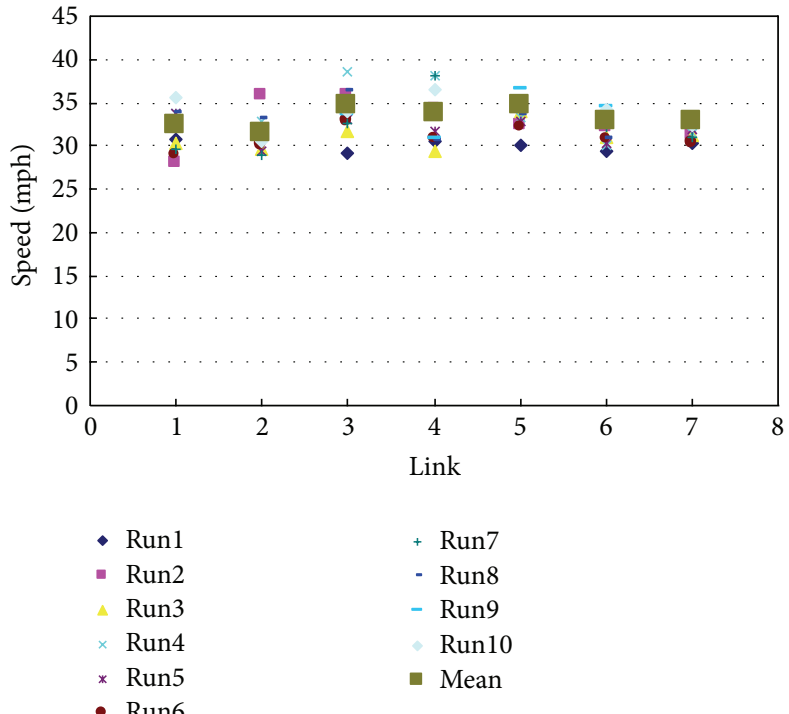

(b) Northbound

FIGURE 8: Average practical travel speeds for links.

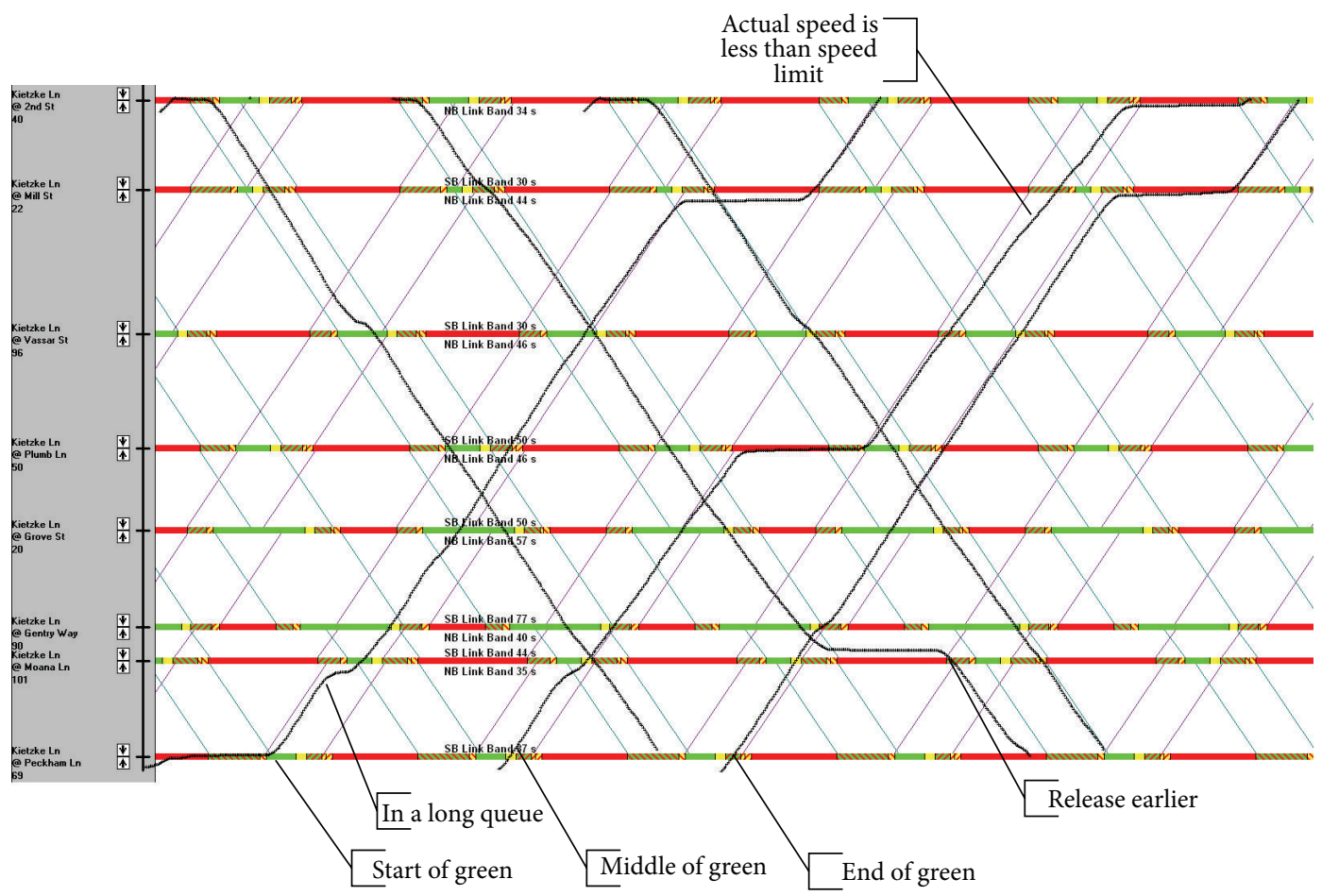

FIGURE 9: GPS trajectory on initial TSD on the speed of $40 \mathrm{mph}$.

average delay and queue length for the nodes, and average delay for the links are the main simulation results, shown in Table 11.

The results show that a rather significant improvement is achieved after the fine-tuning of signal timing plan with practical travel speed data is done.

\section{Conclusion}

Bandwidth can be defined in terms of two consecutive intersections (link bandwidth) or in terms of an entire arterial (arterial bandwidth). In most previous studies, bandwidth is always referred to as arterial bandwidth. In practice, a balance 
TABLE 10: Attainability of link bandwidth on Kietzke Lane with two algorithms.

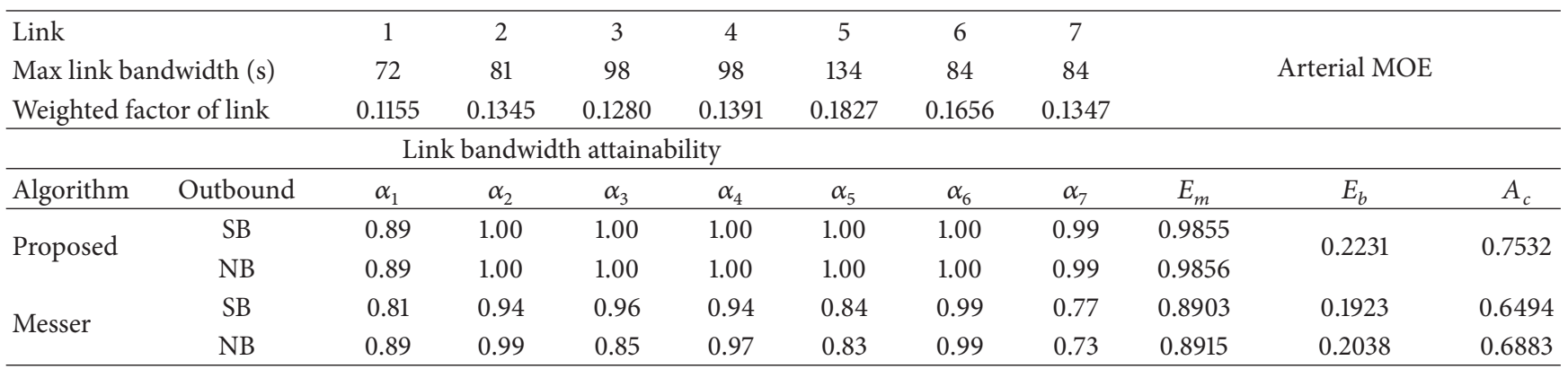

TABle 11: Performance index of signal timing plans with different speeds.

\begin{tabular}{lcccccc}
\hline Type & Index & 32 & Practical speed & 35 & 38 & 40 (speed limit) \\
\hline \multirow{2}{*}{ Network performance } & Average speed (mph) & 18.01 & $\mathbf{1 9 . 1 2}$ & 17.95 & 17.9 & 17.69 \\
& Average delay (s) & 57.25 & $\mathbf{5 3 . 2}$ & 57.91 & 58.29 & 61.83 \\
\hline \multirow{3}{*}{ Nodes } & Total average delay (s) & 210.7 & $\mathbf{1 9 0 . 6}$ & 209.1 & 213.2 & 221.4 \\
& Total average queue (ft) & 519.8 & $\mathbf{5 0 5 . 1}$ & 527.6 & 532.8 & 548.2 \\
& Total intersection control delay & 257.2 & $\mathbf{2 3 5 . 8}$ & 260.6 & 267.2 & 266.2 \\
& Delay from VISSIM & 378.7 & $\mathbf{3 5 0 . 6}$ & 370.3 & 382.1 & 392.7 \\
& Delay from SYNCHRO & 378.3 & $\mathbf{3 6 4 . 3}$ & 382.6 & 397.6 & 401.3 \\
\hline
\end{tabular}

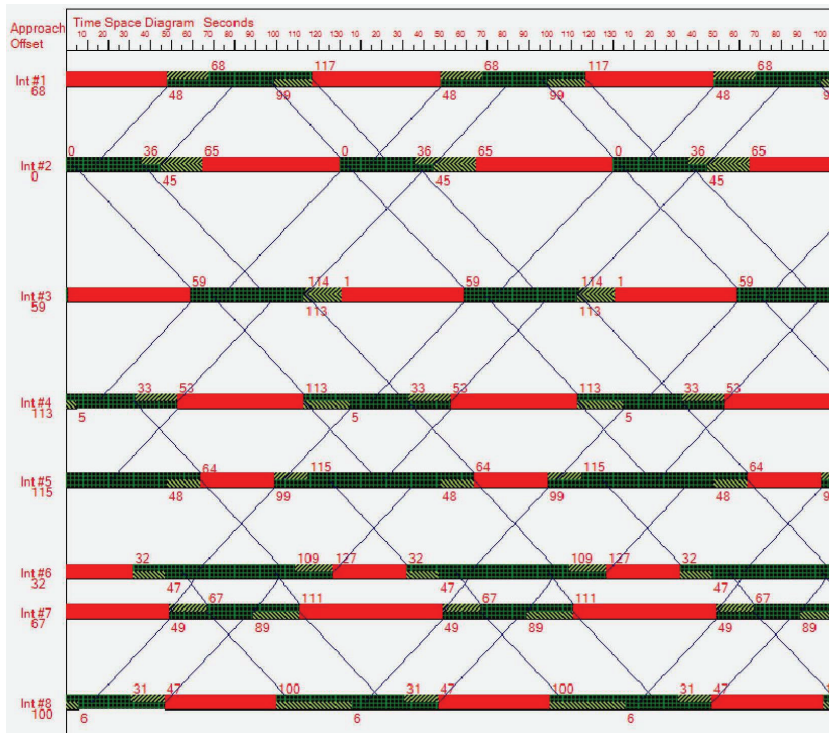

FIGURE 10: TSD of signal timing plan with practical travel speed.

between link bandwidth and arterial bandwidth has proven to be important in optimizing coordinated signal timing plans, because not all drivers need to pass through all the intersections on an arterial. This paper proposes an algorithm on how to obtain an optimal coordinated signal timing plan with both optimized link and arterial bandwidth. The proposed algorithm has two parts: link bandwidth optimization and arterial bandwidth optimization. Link bandwidth attainability is defined as an additional measure of effectiveness for assessing the optimized results. In the link bandwidth optimization, there are eight cases to calculate upper/lower interference and six cases to calculate offset between two consecutive intersections, based on the improvement of Messer's research. The arterial bandwidth optimization has 3-fold processes, such as calculation of link bandwidth, selection of phase sequence, and calculation of offset, generally link by link. Weighted means of link bandwidth attainability is defined as another measure of effectiveness for selecting the best solution of different phase sequences. The results in the case study show that the proposed algorithm can be used to get a much better signal timing plan than that from Messer's algorithm. Additionally when the number of signals in a system increases, it becomes more difficult to obtain a good bandwidth solution with Messer's algorithm. There is no valid solution of arterial bandwidth using Messer's algorithm when the number of signals is very high. However, the proposed algorithm of this paper can be used to obtain an optimal coordinated signal timing plan with both optimal link bandwidth and arterial bandwidth. Fine-tuning of initial signal timing plan is done to improve link and arterial progression bandwidth using practical travel speed compared to speed limit. The evaluation results show that a rather significant improvement is achieved. In future, we will continue our research on optimizing coordinated signal timing plans under the consideration of the traffic volumes of left-turns and earlier release of left-turn phase.

\section{Conflict of Interests}

There is no known conflict of interests associated with this paper and there has been no significant financial support for 
this work that could have influenced its outcome. The authors do not have any possible conflict of interests.

\section{Acknowledgments}

This study is supported by Project "The Fundamental Research Funds for the Central Universities (no. 2013JBM049)" and "National Basic Research Program of China (no. 2012CB725403)."

\section{References}

[1] Federal Highway Administration and USDOT, Traffic Signal Timing Manual, United States Department of Transportation, Washington, DC, USA, 2008.

[2] J. Bonneson, S. Sunkari, and M. Pratt, Traffic Signal Operations Handbooks, Texas Transportation Institute, Texas DOT, Texas, Tex, USA, 2009.

[3] Minnesota DOT, Mn/DOT Traffic Signal Timing and Coordination Manual, Minnesota Department of Transportation, Saint Paul, Minn, USA, 2009.

[4] Z. Tian and T. Urbanik, "System partition technique to improve signal coordination and traffic progression," Journal of Transportation Engineering, vol. 133, no. 2, pp. 119-128, 2007.

[5] X. K. Yang, "Comparison among computer packages in providing timing plans for Iowa arterial in Lawrence, Kansas," Journal of Transportation Engineering, vol. 127, no. 4, pp. 311-318, 2001.

[6] K. G. Baass, "Another look at bandwidth maximization," Transportation Research Record, no. 905, pp. 38-47, 1983.

[7] C. J. Messer, R. H. Whitson, C. Dudek, and E. J. Romano, "A variable -sequence multiphase progression optimization program," Highway Research Record, no. 445, pp. 24-33, 1973.

[8] C. J. Messer, H. E. Haenel, and E. A. Koeppe, "A report on the user's manual for progression analysis and signal system evaluation routine-PASSER II," Research Report 165-14, Texas Transportation Institute, College Station, Tex, USA, 1974.

[9] J. D. C. Little, M. D. Kelson, and N. H. Gartner, "MAXBAND: a program for setting signals on arteries and triangular networks," Transportation Research Record, no. 795, pp. 40-46, 1981.

[10] N. H. Gartner, S. F. Assmann, F. Lasaga, and D. L. Hou, "MULTIBAND: a variable-bandwidth arterial progression scheme," Transportation Research Record, vol. 1287, pp. 212-222, 1990.

[11] C. Stamatiadis and N. H. Gartner, "MULTIBAND-96: a program for variable-bandwidth progression optimization of multiarterial traffic networks," Transportation Research Record, no. 1554, pp. 9-17, 1996.

[12] N. Papola, "Bandwidth maximization: split and unsplit solutions," Transportation Research Part B, vol. 26, no. 5, pp. 341-356, 1992.

[13] N. Papola and G. Fusco, "Maximal bandwidth problems: a new algorithm based on the properties of periodicity of the system," Transportation Research B, vol. 32, no. 4, pp. 277-288, 1998.

[14] H. K. Sripathi, N. H. Gartner, and C. Stamatiadis, "Uniform and variable bandwidth arterial progression schemes," Transportation Research Record, no. 1494, pp. 135-145, 1995.

[15] R. S. Pillai, A. K. Rathi, and S. L. Cohen, "A restricted branchand-bound approach for generating maximum bandwidth signal timing plans for traffic networks," Transportation Research B, vol. 32B, no. 8, pp. 517-529, 1998.
[16] C. Feng, Projection algorithm for improved corridor signal coordination [M.S. thesis], Master of Science in Engineering, University of Akron, Civil Engineering, Akron, Ohio, USA, 2009.

[17] N. H. Gartner and C. Stamatiadis, "Arterial-based control of traffic flow in urban grid networks," Mathematical and Computer Modelling, vol. 35, no. 5-6, pp. 657-671, 2002.

[18] D. Husch and J. Albeck, Synchro 6: Traffic Signal Software, User Guide, Trafficware, Albany, Calif, USA, 2003.

[19] S. Rhyne, "Utilizing GPS and GIS in traffic signal coordination," in Proceedings of the Annual Meeting and Exhibit Compendium of Technical Papers (ITE '05), vol. 8, 2005.

[20] C. A. Quiroga and D. Bullock, "Travel time studies with global positioning and geographic information systems: an integrated methodology," Transportation Research $C$, vol. 6C, no. 1-2, pp. 101-127, 1998.

[21] H. Rakha, F. Dion, and H. Sin, "Using global positioning system data for field evaluation of energy and emission impact of traffic flow improvement projects: issues and proposed solutions," Transportation Research Record, no. 1768, pp. 210-223, 2001.

[22] Z. A. Sabra, Global Positioning System for Time-Based Signal Coordination: Benefit-Cost Analysis, Baltimore Regional Traffic Signal Forum, Baltimore, Md, USA, 2nd edition, 2005.

[23] JAMER Technologies, GPS Travel Time Reference ManualTravel Time and Delay Data Collection Software, JAMER Technologies, Hatfield, Pa, USA, 2004.

[24] JAMER Technologies, PC-Travel for Windows Reference Manual-Travel Time and Delay Analysis Software, JAMER Technologies, Hatfield, Pa, USA, 2004. 


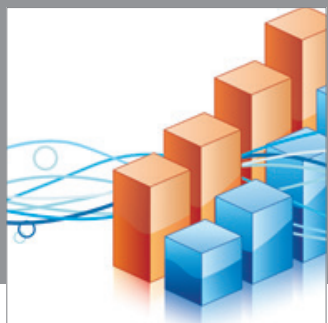

Advances in

Operations Research

mansans

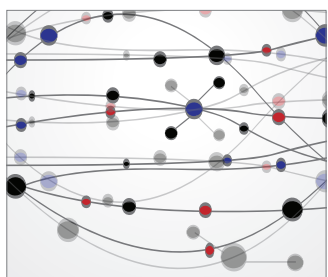

The Scientific World Journal
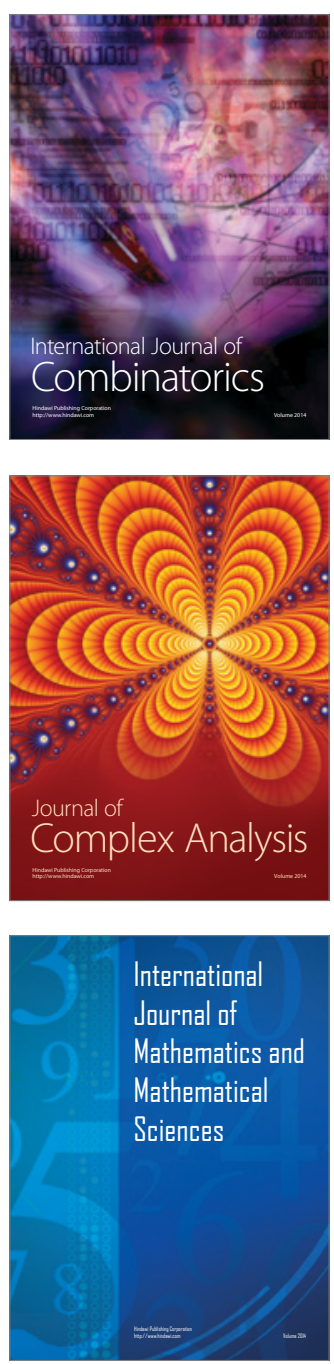
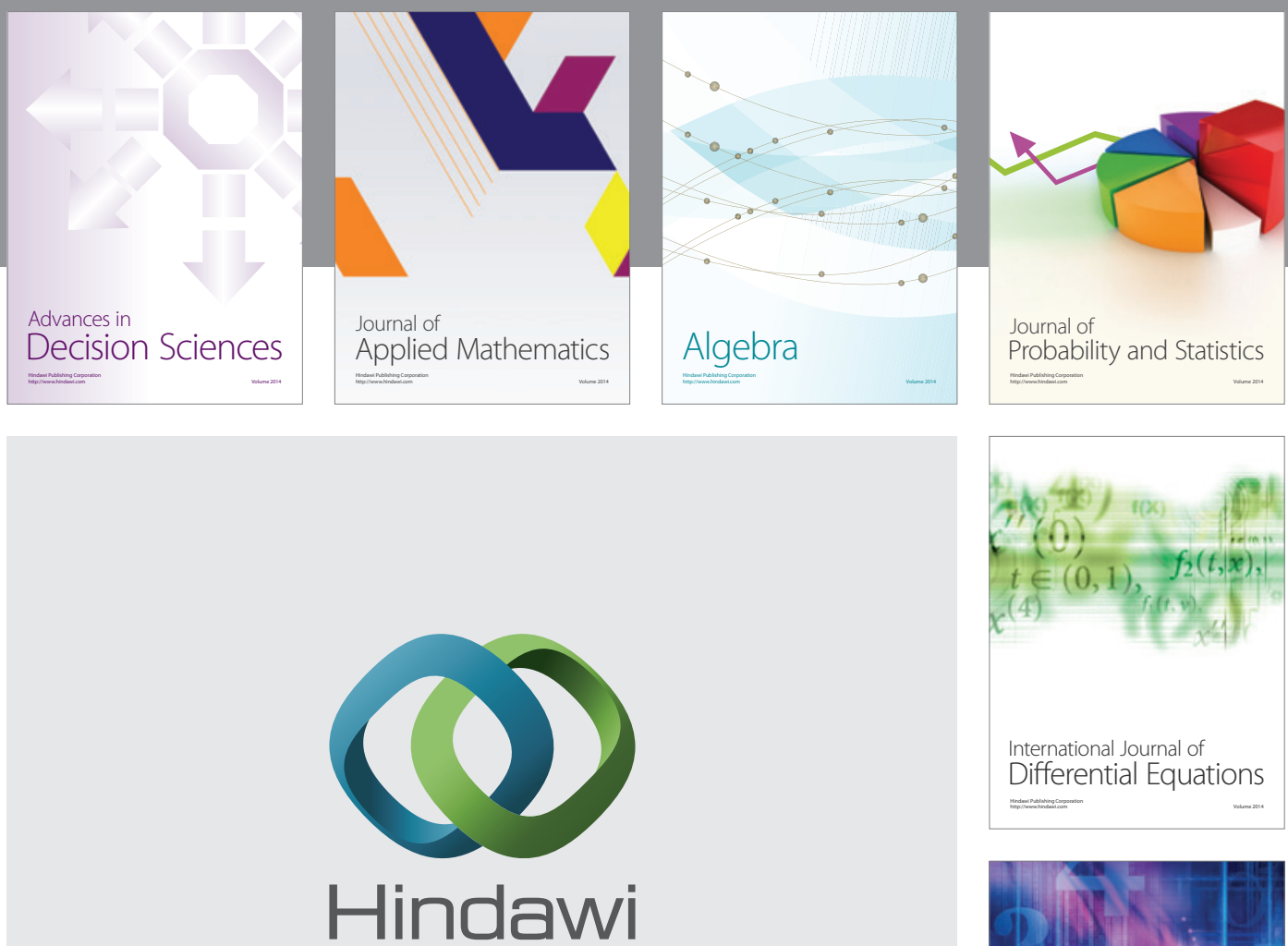

Submit your manuscripts at http://www.hindawi.com
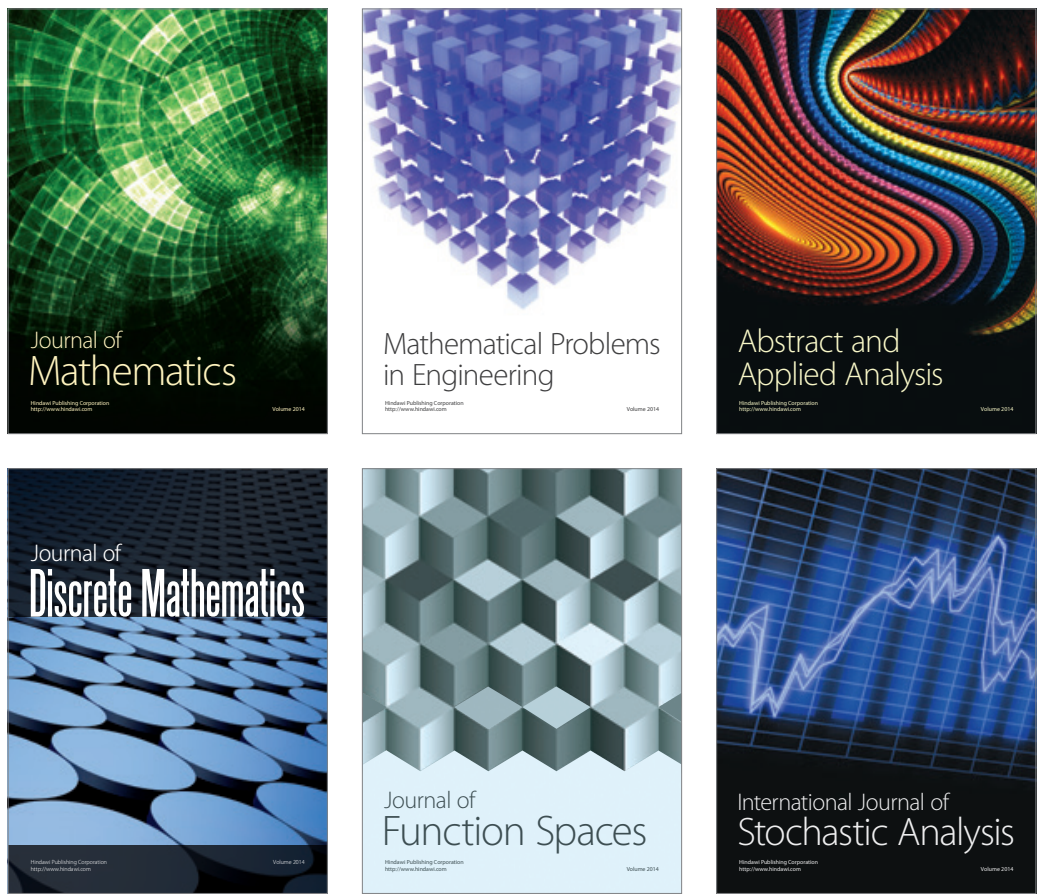

Journal of

Function Spaces

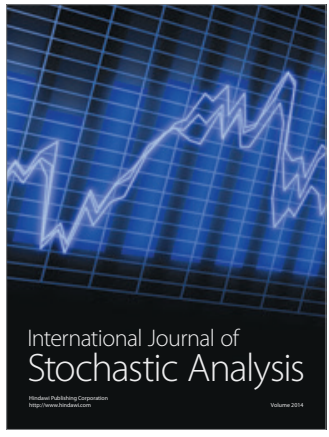

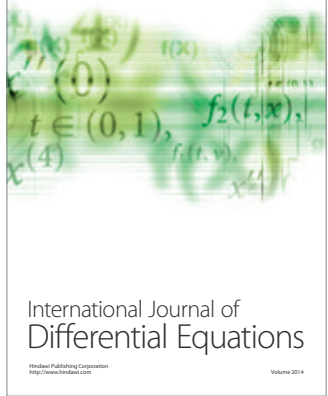
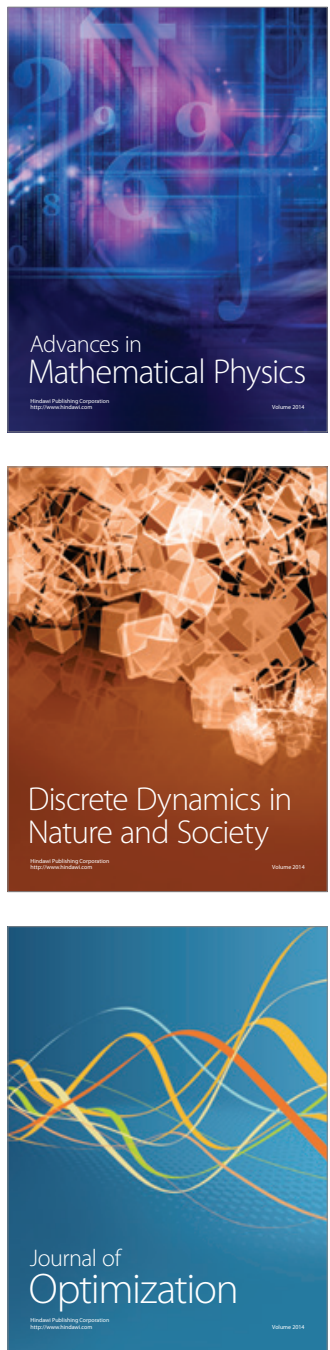